\title{
ON PRECOMPACT (QUASI-) UNIFORM STRUCTURES
}

\author{
P. TH. LAMBRINOS
}

ABSTRaCt. The following are shown:

(1) The subbasis theorem on precompactness does not hold even in uniform spaces.

(2) The supremum of (even finitely many) precompact quasi-uniform structures is not necessarily precompact.

(3) A compact quasi-uniform space is not necessarily totally bounded.

These results contradict corresponding assertions in the literature.

1. Introduction. By definition, a quasi-uniform space is a space fulfilling the axioms of a uniform space with the possible exception of the symmetry axiom.

Each topological space may be considered as a quasi-uniform space and vice versa.

Two of the most useful notions in such spaces are those of "total boundedness" and "precompactness". A (quasi-) uniform space $(X, \mathcal{Q})$ is called totally bounded iff for each entourage $V \in \mathcal{Q}$ there exists a finite family of subsets $A_{1}, \ldots, A_{n}$ of $X$ such that

$$
\cup\left\{A_{k}: k=1, \ldots, n\right\}=X \text { and } \cup\left\{A_{k} \times A_{k}: k=1, \ldots, n\right\} \subset V
$$

$(X, \mathcal{Q})$ is called precompact iff for each entourage $V \in \mathcal{Q}$ there exists a finite subset $\left\{x_{1}, \ldots, x_{p}\right\}$ of $X$ such that $\cup\left\{V\left(x_{k}\right): k=1, \ldots, p\right\}=X$.

A uniform space is totally bounded iff it is precompact. A totally bounded quasi-uniform space is precompact, but the converse does not hold, in general.

The above results may be found for example, in [2], [3].

2. Remarks on subbase and supremum of (quasi-) uniform structures. According to the subbase theorems [2, p. 205], [3, p. 49] in the above given definition of total boundedness in a (quasi-) uniform space $(X, Q)$, we may replace the part "for each entourage $V \in \mathcal{Q}$ " by the expression "for each entourage $V$ of a given subbase $\mathcal{U}_{s}$ of the (quasi)-uniformity $\mathscr{U}$ ".

[3, p. 49] and [1, Lemma 3, p. 302] assert that similar subbase theorems hold also in precompact (quasi)-uniform spaces. This, however, is not true in

Received by the editors July 28, 1975 and, in revised form, December 8, 1975.

AMS (MOS) subject classifications (1970). Primary 54E15.

Key words and phrases. Precompact (quasi-) uniform structures. 
general, even in the case of uniform spaces, as the following counterexample shows: 1

Let $X$ be the set of the reals. Then, the family $\mathscr{Q}_{s}=\{V, W\}$, where $V=\{(x, y): x, y \leqslant a$ or $x, y \geqslant a\}, W=\{(x, y): x=y$ or $x<a<y$ or $y$ $\langle a<x\}, a \in R$, is a subbase for the discrete uniformity on $X$, which, of course, is not precompact since it contains the diagonal. Nevertheless, if we take $t_{1}<a<t_{2}$, then $V\left(t_{1}, t_{2}\right)=X=W\left(t_{1}, a, t_{2}\right)$.

In fact, the equality $\left(S_{1} \cap \cdots \cap S_{m}\right)(A)=S_{1}(A) \cap \cdots \cap S_{m}(A)$ used in [1, Lemma 3, p. 302] does not hold in general (see the above counterexample). However, although this false equality has been also used in [1, Lemma 4, p. 303] its result that "the least upper bound of precompact uniform structures is precompact" is really true, since for uniform structures precompactness is equivalent to total boundedness and the subbasis theorem [2, p. 205] holds for total boundedness. On the other hand, the corresponding proposition on quasi-uniform structures is not true in general, as the counterexample below shows (thus contradicting Theorem 4.10.vi.b of [3, p. 50]).

Let $X$ be as before; then the set $V_{a, b}=\{(x, y): x=y$ or $a<x<b\}$, where $a, b \in X, a<b$, is a base for a precompact quasi-uniformity $\mathscr{2}_{a, b}$ on $X$. However, the supremum of the family $\left\{2_{0,1}, \mathscr{2}_{1,2}\right\}$ is the discrete uniformity which is not precompact. We note that any of the above quasi-uniform spaces $\left(X, \mathscr{2}_{a, b}\right)$ is compact but not totally bounded. Thus, the assertion of [4, p. 368] that "every compact quasi-uniform space is totally bounded" is not true.

Finally, we note that despite the above results, the product theorem on precompact quasi-uniform structures [3, Theorem 4.10.iv.b, p. 50] is true. This can be shown using the fact that a quasi-uniform space is precompact if and only if every ultrafilter in the space is a Cauchy filter [5, Theorem 1.1, p. 79].

\section{REFERENCES}

1. S. Gaal, Point set topology, Academic Press, New York and London, 1964. MR 30 \# 1484.

2. J. L. Kelley, General topology, Van Nostrand, Princeton, N.J., 1955. MR 16, 1136.

3. M. G. Murdeshwar and S. A. Naimpally, Quasi-uniform topological spaces, Noordhoff, Groningen, 1966. MR 35 \#2267.

4. M. G. Murdeshwar and K. K. Theckedath, Boundedness in a quasi-uniform space, Canad. Math. Bull. 13 (1970), 367-370. MR 42 \# 5222.

5. J. L. Sieber and W. J. Pervin, Completeness in quasi-uniform spaces, Math. Ann. 158 (1965), 79-81. MR 30 \# 2449.

Department of Mathematics, University of Thessaloniki, Thessaloniki, Greece

Mathematical Institute, University OF Oxford, 24-9 St. Giles, OXford, England

Current address: Department of Mathematics, Kansas State University, Manhattan, Kansas 66506

1 Meanwhile, the author has been informed that a somewhat similar counterexample has been constructed by Earl Ray McMahan in his unpublished Master's thesis, Completions and compactifications of uniform structures, Southern Illinois University, Carbondale, Illinois, 1966. 